\title{
Les produits d'origine entre cultures et règlements
}

Laurence BÉRARD

Philippe MARCHENAY

CNRS,

Alimentec,

Rue Henri de Boissieu,

01060 Bourg-en-Bresse cedex 09

<laurence.berard@ethno-terroirs.cnrs.fr>

\section{Quand le local fait sens}

De nombreux inventaires, enquêtes de terrain, monographies montrent l'extrême diversité que recouvrent les productions localisées. Elles concernent tous les secteurs de l'agroalimentaire, du végétal à l'animal - fruits et légumes, viandes, volailles, produits de la pêche - en passant par les produits transformés comme les charcuteries, fromages, boulangeriespâtisseries, huiles ou boissons fermentées. Ces productions n'occupent pas toutes la même place dans les communautés qui les ont fait naître. Parfois, c'est le riche soubassement $d^{\prime}$ 'une culture qui émerge derrière un produit. C'est le cas pour les fromages Comté ou Abondance, qui mettent en perspective un système d'élevage, un milieu naturel contraignant, une organisation sociale et des pratiques fromagères spécifiques. Le bœuf charolais détermine le paysage et l'économie de toute une région, son élevage associe une gestion subtile des herbages, des connaissances en matière de sélection, d'engraissement, d'identification des stades de croissance aboutissant à une viande goûteuse. Parfois, le fil est beaucoup plus ténu, comme le montre l'huile de colza grillé dans le nord de l'Ardèche, dont l'originalité tient au seul fait de griller les graines avant de les presser. II n'empêche que nulle part ailleurs, ou presque, on ne retrouve cette huile au goût si particulier. Ce constat de variabilité s'impose aussi au regard des volumes, échelles et zones de production. Les modes de commercialisation et les réseaux de distribution vont de la vente directe à la grande distribution.

Comment raisonner une telle diversité ? Deux critères permettent d'englober l'ensemble de ces productions liées à un lieu : la profondeur historique et les savoir-faire partagés. Ce plus petit dénominateur commun permet d'organiser et de penser cette famille en donnant une première définition de l'origine. Au-delà de cette grande polymorphie, ces productions alimentaires entretiennent également une relation particulière à l'espace. Leur inscription en

\begin{abstract}
Localized agri-food products stand at the crossroads of multiple converging interests that attract an ever-wider audience. The regulations protecting origin-based products raise complex questions regarding notions of tradition, place, origin, reputation or customs. Plainly however, the survival of such products depends on appropriate standards particularly in terms of sanitary requirements - that reflect the specific characteristics of local production systems and small-scale manufacturing units.
\end{abstract}

Key words: origin based products, sanitary requirements, local knowledge, geographical indications

un lieu s'assortit d'une antériorité et de pratiques collectives. En d'autres termes, elles croisent l'espace, le temps et font l'objet de savoirfaire partagés. Ces trois critères sont étroitement imbriqués, le fil directeur étant les pratiques qui relient ancrage historique et relation au lieu. Leur dimension collective les inscrit dans la culture locale et permet de distinguer la provenance (venir d'un lieu), de l'origine (être de ce lieu). L'amplitude temporelle peut être variable suivant les cas, s'échelonner sur plusieurs siècles ou quelques décennies selon l'histoire locale; mais l'antériorité qui donne de l'épaisseur au lieu existe bien, liée à la mémoire collective transmise. Ainsi, les critères d'ordre culturel associant un lieu à une histoire et à un groupe social permettent d'organiser et de penser cette diversité. Cette première grille de lecture regroupe l'ensemble des produits pour lesquels le lieu fait sens et permet de faire un premier tri, en particulier dans le cas des « produits de terroir ». Cette expression, communément utilisée en France, est perturbante car elle confond origine et provenance. La situation est la même pour les produits fermiers, assimilés la plupart du temps à des produits de terroir. Or, ils n'ont d'autre particularité que d'être élaborés à l'échelle de l'exploitation agricole et à partir des matières premières qu'elle fournit. Ils peuvent ou non entrer dans la catégorie des productions localisées selon qu'ils renvoient ou non à la culture locale. Un foie gras fabriqué à la ferme en Normandie est un produit fermier ; dans le Périgord, c'est une production localisée. C'est la raison pour laquelle nous préférons employer le vocable « production localisée ».

En aval de la fabrication, les modes d'utilisation occupent une place importante. Ils ont trait tant à la préparation des aliments qu'à la façon de les accommoder ou aux associations qu'ils privilégient. La consommation, à l'instar du processus de production, est un phénomène localisé qui passe par une grande familiarité entre le consommateur et le produit. La culture alimentaire se différencie d'un pays à l'autre, mais aussi entre régions et zones géographi- ques d'un même pays. La connaissance des caractéristiques du produit, les compétences dans la préparation et dans l'utilisation, la liaison avec des moments et des valeurs culturelles partagées en un lieu, sont autant d'éléments qui forment le cadre de la consommation locale.

Une différence marquée s'observe entre les consommateurs locaux qui savent de quoi ils parlent et ce qu'ils recherchent, et les autres, dont l'intérêt interfère pour beaucoup avec les grandes préoccupations de notre société. La mondialisation, en entraînant la standardisation des modes de vie, génère paradoxalement un intérêt accru pour le local. C'est ce qui ressort du succès persistant que remportent les marques de distributeur terroirs telles que «Reflets de France » ou « Nos régions ont du talent», très rentables, qui continuent de s'étoffer en élargissant leur gamme.

\section{La protection de l'origine}

La protection de l'indication géographique est une démarche volontaire qui protège le nom d'un produit auquel l'origine confère des qualités particulières. Cette mise en relation d'un lien au terroir et de savoir-faire spécifiques existe depuis longtemps en France.

La mise en place d'une réglementation reprenant et élargissant ce dispositif à l'échelle européenne - à travers I'Appellation d'origine protégée et l'Indication géographique protégée n'a fait que renforcer l'attention portée aujourd'hui aux productions localisées. La philosophie de I'AOP est de protéger, à travers un nom, un produit unique et non reproductible dans un autre terroir. L'ensemble du processus de production doit se faire dans une seule et même zone dont il faut démontrer la cohérence et l'influence vis-à-vis des caractéristiques du produit.

L'IGP, qui protège aussi un nom géographique, se fonde plutôt sur la réputation du produit, sur son histoire, liée à celle d'une localité et sur des caractéristiques ou des qualités particulières. 
Elle n'impose pas une zone unique où doit se dérouler l'ensemble des opérations : les matières premières en particulier peuvent provenir d'ailleurs.

L'histoire joue un rôle important dans la caractérisation du lien à un lieu. En tant que discipline, elle a souvent du mal à rendre compte de la profondeur historique de ces produits. En effet, les témoignages sur lesquels s'appuyer pour l'étude de ces productions sont en partie assujettis aux préférences alimentaires des puissants de l'époque, ce qui laisse dans l'ombre, voire élimine une bonne partie d'entre elles. Si excellent que soit un fromage ou un jambon, il demeurera anonyme si sa consommation reste circonscrite au cadre étroit de la communaute qui le produit. Par ailleurs, bon nombre de ces productions locales étaient élaborées à I'échelle domestique, dans une logique strictement autarcique. De ce fait, elles ont rarement laissé de traces écrites de leur présence à travers les siècles. Pour toutes ces raisons, I'histoire orale semble bien appropriée pour compléter efficacement les données obtenues, voire pour constituer dans un certain nombre de cas l'essentiel de l'information. L'absence d'archives écrites ne veut pas dire pour autant que les produits n'ont pas d'ancrage historique.

Les savoir-faire partagés constituent une autre caractéristique forte des productions locales. Ils peuvent avoir trait à l'élevage ou la culture, à la sélection d'une race, à un type de caillage, d'affinage, à toutes pratiques liées à l'élaboration d'un produit, à tout usage de consommation qui contribue à l'ancrer dans une culture locale et à le définir.

Comment les identifier et les prendre en compte dans une démarche de protection? L'élaboration d'un cahier des charges, à travers la mise à plat des pratiques, des savoirs qui leur sont associés, des définitions, met en lumière toutes les difficultés inhérentes à une codification de la culture technique locale.

En filigrane de la caractérisation des savoir-faire locaux et des relations qu'ils entretiennent avec les savoirs techniques et scientifiques, se pose la question de la tradition, associée à la transmission. En effet, jusqu'à quel point un usage et avec lui un paramètre de production peut-il évoluer? Quels sont les éléments qui constituent le noyau dur d'une tradition, sur lesquels on ne peut intervenir sous peine de remettre en question son existence même? Mais à quo renvoie plus précisément ce terme qui semble tellement aller de soi que l'on se garde bien de le définir?

Contrairement à la représentation que s'en font nos cultures, la tradition n'est pas une survivance du passé dans le présent, le legs encore vivant d'une époque globalement révolue, mais un «point de vue » que les hommes du présent développent sur ce qui les a précé- dés, une interprétation du passé conduite en fonction de critères rigoureusement contemporains. Ce n'est pas le passé qui produit le présent mais le présent qui façonne son passé. Cette lecture sélective du passé selon des critères culturellement significatifs peut être mise en perspective avec l'évolution des usages et avec l'acte de transmettre.

Les usages et la tradition relèvent en partie de la même problématique: que choisit-on de léguer? Chaque syndicat de défense se trouve confronté aux mêmes difficultés dans le cadre de la conception du cahier des charges ou de sa modification, dès lors qu'il s'agit de consigner dans le détail les différentes informations sur l'élaboration du produit. Les choix diffèrent d'un produit à l'autre, renvoyant à la place qu'il occupe, à la dimension identitaire plus ou moins prononcée qu'il recouvre, au rôle qu'on veut lui faire jouer. Toutes ces questions se posent aujourd'hui avec beaucoup d'acuité ; elles doivent continuer à être débattues par les détenteurs de ces savoirs transmis, réinterprétés, modifiés au fil des générations, savoirs qui constituent une forme de propriété intellectuelle collective.

La loi d'orientation agricole du 5 janvier 2006 modifie en profondeur un certain nombre de points concernant le dispositif français en particulier les contrôles. Avant 2006, I'AOP européenne était assimilée en France à l'AOC et gérée selon les mêmes règles. L'Institut national des appellations d'origine ${ }^{1}$, établissement public, partageait avec la DGCCRF (Direction générale de la concurrence, de la consommation et de la répression des fraudes) la responsabilité des contrôles. Le système de contrôle des signes d'identification de la qualité et de I'origine est aujourd'hui réformé et tous les produits, y compris les $\mathrm{AOC}$, doivent dorénavant être contrôlés par des organismes indépendants des producteurs, agréés par l'INAO. Les filières de faible importance et les petits producteurs pourront-ils assumer les charges financières induites par le coût des contrôles externes?

Cette réglementation se traduit dans un certain nombre de cas par des réussites remarquables en matière de valorisation des productions locales. Toutefois, elle peine souvent à protéger ce qui fait réellement la spécificité de ces productions. Par ailleurs celles-ci sont d'une certaine façon victime de leur succès auprès des consommateurs. L'industrie agroalimentaire a investi ce créneau porteur, en particulier en ce qui concerne le secteur fromager; elle tend à banaliser les modes de production quand ce n'est pas la déstabilisation de la filière tout entière, comme c'est parfois le cas.

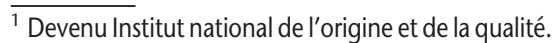

\section{Les normes sanitaires}

La question des normes sanitaires apparaît fréquemment en filigrane dans le discours des producteurs $^{2}$. Le coût de mise aux normes a souvent des conséquences graves pour l'avenir de certaines entreprises. De multiples petites fromageries ferment tout simplement leurs portes. On observe une diminution régulière de la fabrication en alpage liée à la difficulté qu'ont les éleveurs à respecter des normes trop contraignantes. Nombre d'entre eux cessent leur activité et vendent leur lait, comme c'est le cas en abondance. Chez les artisans, beaucoup de charcutiers se maintiennent bon an mal an s'ils n'ont pas de sérieuses motivations pour "se mettre aux normes ». Ils ne réalisent pas les travaux nécessaires, les jugeant trop coûteux et ne trouvent pas de repreneurs lorsqu'ils arrivent à la retraite, ce qui constitue une perte pour le tissu économique et la culture technique. Par ailleurs, les mesures d'hygiène imposées peuvent perturber les processus de fermentation et d'affinage, qui continuent d'être les clefs de voûte de la spécificité pour une grande partie des productions traditionnelles. Les aménagements exigés au titre de la mise aux normes transforment parfois complètement l'écologie microbienne des lieux et peuvent entraîner de grosses difficultés pour les fabrications.

Les réglementations d'hygiène ont longtemps été formulées pour la grande industrie sans prendre en compte les petites unités. II semblerait que les choses évoluent. Une nouvelle réglementation européenne sur la sécurité et I'hygiène des aliments, communément appelée "Paquet hygiène ", est entrée en vigueur le 1er janvier 2005. L'objectif général de cette refonte des textes précédents est d'harmoniser et simplifier la réglementation européenne ainsi que les systèmes de contrôles nationaux, d'avoir une approche intégrée de toute la filière, de la production primaire jusqu'à la table du consommateur. L'Union européenne s'est ainsi dotée d'une politique unique en matière $d^{\prime}$ hygiène et de gestion de la sécurité alimentaire, applicable à tous les maillons du secteur alimentaire et visant à faciliter la libre circulation des denrées. Les notions de responsabilité, traçabilité, analyse des risques, principe de précaution se situent au cœur de ces préoccupations. La procédure fondée sur le principe de I'HACCP ${ }^{3}$, qui repose sur l'analyse des risques et la maîtrise des points critiques, est généralisée et l'élaboration de guides de bonnes pratiques par les branches profession-

\footnotetext{
$\overline{2}$ Voir à ce sujet Ricard, 1998, Produits de terroir et normes de fabrication. Les fromages AOC face aux nouvelles exigences sanitaires, p. 103-14.

${ }^{3}$ Hazard analysis critical control points.
} 
nelles des secteurs concernés est fortement encouragée. Les concepts et procédures sont dictés par des principes relatifs à la protection de la santé et à la sécurité du consommateur. La Commission européenne est consciente des questions soulevées par ce nouveau cadre réglementaire appliqué à l'ensemble du secteur alimentaire. Dans I'introduction aux deux règlements 852 et $853^{4}$, elle affirme la nécessité "de prévoir une certaine souplesse de manière à permettre de poursuivre l'utilisation de méthodes traditionnelles à tous les stades de la production, de la transformation ou de la distribution des denrées alimentaires et à l'égard des exigences structurelles imposées aux établissements. " Ces dérogations relèvent de deux registres différents: locaux, équipement et matériaux pour les unes; pratiques et savoirfaire pour les autres. Les demandes de dérogation font l'objet de procédures complexes dans le cadre de l'application française du règlement. Les producteurs, par I'intermédiaire des interprofessions, doivent faire la preuve de la non-dangerosité de leurs pratiques; toutefois la volonté affichée de prendre en compte les productions traditionnelles constitue une avancée non négligeable.

La distorsion reste grande entre les discours sur la dimension patrimoniale de ces productions, le rôle qu'elles sont susceptibles de jouer dans le développement local et la réalité du terrain.

\footnotetext{
${ }^{4}$ Considérants 16 du 852 et 19 du 853.
}

Ces productions continuent d'être menacées pour certaines d'entre elles et pérenniser leur spécificité reste un défi dans le contexte réglementaire actuel.

\section{RÉFÉRENCES}

1. ALPHANDERY $P$, BITOUN $P$, DUPONT $Y$. L'équivoque écologique. Paris: La Découverte, 1992.

2. AUGE M. Pour une anthropologie des mondes contemporains. Paris : Aubier, 1994.

3. BERANGER C, VALCESCHINI E. (dir.). Qualité des produits liée à leur origine. Actes. Séminaires INRA. 10 et 11 décembre 1998. Paris: INRA, 1999.

4. BERARD L, FROC J, HYMAN P, HYMAN M, MARCHENAY P. 1992 - 1996 - Inventaire du patrimoine culinaire de la France. Paris, Albin Michel, (Nord-Pas-de-Calais, 1992; Ile-deFrance, 1993 ; Bourgogne, 1993 ; FrancheComté, 1993 ; Pays de la Loire, 1993 ; PoitouCharentes, 1994 ; Bretagne, 1994 ; RhôneAlpes, 1995 ; Provence-Alpes-Côte d'Azur, 1995 ; Midi-Pyrénées, 1996 ; Corse, 1996; Aquitaine. 1997).

5. BERARD L, MARCHENAY PH. Les produits de terroir entre cultures et règlements. Paris: CNRS éditions, 2004.

6. CHEVALLIER D. Savoir faire et pouvoir transmettre. Transmission et apprentissage des savoir-faire et des techniques. Paris: Editions de la Maison des Sciences de I'Homme, 1991.
7. CNRS, Chambre régionale d'agriculture de Rhône-Alpes, ISARA, PEA CRITT. L'application du Paquet Hygiène aux produits traditionnels. 2007 ; (Fiche technique, 4 p).

8. FISCHLER C. L'Homnivore. Paris: Odile Jacob, 1990.

9. FROC J, HYMAN P, HYMAN M. 1996-2000 Inventaire du patrimoine culinaire de la France, Paris, Albin Michel, (Martinique, ChampagneArdennes, 2000 ; Guyane, 1999; Picardie, 1999 ; Alsace, 1998; Guadeloupe,1998; Languedoc-Roussillon, 1998 ; Limousin, 1998, Lorraine, 1998 ; Martinique. 1997).

10. LENCLUD G. Qu'est-ce que la tradition? In : Detienne M, ed. Transcrire les mythologies. Tradition, écriture, historicité. Paris: Albin Michel, 1994 : 25-44

11. MOITY-MAÏZI P, SAINTE-MARIE C, GESLIN P, MUCHNIK I, SAUTIER D. Systèmes agroalimentaires localisés. Terroirs, savoir-faire, innovations. Etudes et recherches sur les systèmes agraires et le développement. Paris : INRA, 2001 ; (32).

12. PILLEBOUE J. La qualité agro-alimentaire et ses territoires. Sud-Ouest Européen. Toulouse : Presses Universitaires du Mirail, PUM, 1999 ; (6).

13. RICARD D. Produits de terroir et normes de fabrication. Les fromages AOC face aux nouvelles exigences sanitaires. In : Bérard L, Marchenay $\mathrm{P}$, eds. Patrimoine, montagne et biodiversité. Rev. Geogr. Alp. 1998 : 103-15 ; (4/86). 